\title{
Functional genomics in translational cancer research: focus on breast cancer
}

Isik G. Yulug and Bala Gur-Dedeoglu

Advance Access publication date 7 March 2008

\begin{abstract}
Conventional molecular and genetic methods for studying cancer are limited to the analysis of one locus at a time. A cluster of genes that are regulated together can be identified by DNA microarray, and the functional relationships can uncover new aspects of cancer biology. Breast cancer can be used to provide a model to demonstrate the current approaches to the molecular analysis of cancer. Meta-analysis is an important tool for the identification and validation of differentially expressed genes to increase power in clinical and biological studies across different sets of data. Recently, meta-analysis approaches have been applied to large collections of microarray datasets to investigate molecular commonalities of multiple cancer types not only to find the common molecular pathways in tumour development but also to compare the individual datasets to other cancer datasets to identify new sets of genes. Several investigators agree that microarray results should be validated. One commonly used method is quantitative reverse transcription PCR (qRT-PCR) to validate the expression profiles of the target genes obtained through microarray experiments. qRT-PCR is attractive for clinical use, since it can be automated and performed on fresh or archived formalin-fixed, paraffin-embedded tissue samples. The outcome of these analyses might accelerate the application of basic research findings into daily clinical practice through translational research and may have an impact on foreseeing the clinical outcome, predicting tumour response to specific therapy, identification of new prognostic biomarkers, discovering targets for the development of novel therapies and providing further insights into tumour biology.
\end{abstract}

Keywords: breast cancer; gene expression; microarrays; functional genomics; meta-analysis; qRT-PCR

\section{INTRODUCTION}

Components and behaviours of biological systems can be studied using the many tools of genomics, such as SNPs [1, 2], CGH [3], SSH [4], SAGE [5, 6], proteomics [7] and siRNA technology [8]. It is widely believed that functional genomics will transform our understanding of the mechanisms underlying cellular function, and in combination with bioinformatics promises to accelerate the application of basic discoveries into clinical practice despite the natural cautions associated with the implementation of new technologies in the clinical arena.

The human genome sequence is now available and we have started to understand genomic complexity at the DNA and gene expression levels. The development of new technologies for the large-scale analysis of the genome, transcriptome, proteome and metabolome has enabled functional genomics to have a profound impact on clinical medicine $[9,10]$. An astounding amount of molecular data resulting from rapid usage of these techniques have accumulated and a multitude of sophisticated methods and algorithms have been developed for comprehensive analysis of these data [11].

The application of genomics technologies to the study of cancer is rapidly shifting toward the analysis of clinically relevant samples derived from patients to discover new biomarkers for early detection of cancers. Since characteristic patterns of gene expression can be measured in parallel by using microarrays, gene expression profiling with DNA microarrays has emerged as a powerful approach to study the transcriptome of individual cancers. Molecular biologists work with clinicians and pathologists to obtain

Corresponding author. Isik G. Yulug, PhD, Faculty of Science, Department of Molecular Biology and Genetics, Bilkent University, Ankara 06800, Turkey. Tel: +90-312-290-2506; Fax: +90-312-266-5097; E-mail: yulug@fen.bilkent.edu.tr

Isik G. Yulug is an Associate Professor at Bilkent University, Department of Molecular Biology and Genetics. Her main area of research is molecular genetics of breast cancer and expression profiling.

Bala Gur-Dedeoglu is a senior PhD student and works with Dr Yulug in breast cancer gene expression profiling.

(C) The Author 2008. Published by Oxford University Press. For permissions, please email: journals.permissions@oxfordjournals.org 
samples from patients with a known medical history, so that the molecular characteristics of samples can be correlated with the clinical presentation. This approach provides an insight into molecular mechanisms of the different cancer types, and also helps to find novel cancer biomarkers.

There are several published studies that highlight the remarkable impact of DNA microarrays on cancer research [12-18]. For example, gene expression signatures for the major cancer types and the correlation with various tumour characteristics that determine tumour grade or differentiation, metastasis and survival have been identified through these studies [19-22].

\section{MOLECULAR PROFILING OF BREAST CANCER}

Breast cancer is a major problem in developed countries and the different classifications of this disease are mostly based on clinical and pathological factors, which unfortunately fail to reflect the heterogeneity of the tumours. There are some histological markers available to decide on the prognosis and treatment of breast cancer. Estrogen receptor (ER) status, as ER-positive or ER-negative, helps to categorize breast cancers into two major classes. ERBB2 (Her-2/Neu) is also routinely used to classify breast cancer into HER-2 amplified or non-amplified categories. There are other single gene markers such as TP53, and cell proliferation markers such as Ki-67, and cyclin D1 that have emerged from detailed molecular analysis [23].

While conventional methods were restricted to studying a single locus, current high-throughput techniques have allowed monitoring gene expression or copy number levels of almost all known genes in a single experiment. Molecular profiling has been shown to be well-suited to phenotypic characterization of breast cancer and potentially to discover new molecular classes among cancers with similar histopathological appearance [24-29]. Several landmark microarray studies have demonstrated that one can build a molecular taxonomy of breast tumours using this technology and can provide a more sophisticated molecular picture together with individualized recurrence risks.

\section{Distinguishing tumours on the basis of their gene expression profiles: impact on the future of breast cancer research}

Gene expression profiling using DNA microarrays has provided an opportunity to perform more detailed and individualized breast tumour characterization leading to classification of breast cancer into distinct new molecular subgroups [30]. The potential advantages of improving tumour classification by expression profiling has been central to several large-scale breast cancer studies over the past few years that have reported identification of signature gene lists with potential for prediction of clinical outcome [24, 25, 29, 31, 32].

One of the first comprehensive studies classifying sporadic breast tumours into subtypes distinguished by differences in their expression profiles was performed by Perou et al. [33]. Using 40 tumours and 20 matched pairs of samples they identified an 'intrinsic gene set' of 476 cDNAs and then used this to cluster and segregate the tumours into four major subgroups: a 'luminal-like cells' group expressing ER; a 'basal-like cells' group expressing cytokeratins 5 and 17, integrin 4 and laminin, but lacking ER expression; an 'ERBB2-positive' group, and a 'normal like' epithelial group [33]. Subsequent studies confirmed that there are large-scale gene expression differences between ER-positive (mostly luminal-like) and ER-negative (mostly basal-like) cancers and suggested that further molecular subsets also exist $[28,34,35]$. The prognosis and chemotherapy sensitivity of the different subgroups are different. The luminal type cancers tend to have the most favourable long-term survival, whereas basallike and ERBB2-positive tumours are more sensitive to chemotherapy [24, 36].

van't Veer et al. have used DNA microarray analysis on the primary breast tumours of 78 lymph node-negative young patients and compared the expression profiles of 34 patients who developed distant metastasis within 5 years and 44 patients who remained disease-free for at least 5 years [25]. Their analysis led to the identification of a 70-gene expression signature that was developed to classify tumours into the good and poor prognosis groups. The results were later confirmed in a larger set of tumours [26, 37]. The genes significantly up-regulated in the poor prognosis signature included those involved in cell cycle, invasion and metastasis, angiogenesis and signal transduction. This 70-gene marker set is now commercially available on the MammaPrint array (Agendia BV, Amsterdam, The Netherlands) and the prospective MINDACT clinical trial is underway to evaluate whether use of the 70-gene classifier is associated with clinical benefit. 
Wang et al. reported a promising study showing the use of DNA microarray data for improving risk assessment for patients with lymph node-negative breast cancer. The investigators identified a diagnostic test based on expression values from a set of 76 genes. They specified 76 genes $(60$ genes for ER-positive, 16 for ER-negative breast tumours) that distinguished lymph node-negative patients who developed distant metastasis within 5 years [38]. The genes included in this prognostic signature belong to many functional classes, including transcriptional regulation, immune response, cell death, cell cycle, growth and proliferation, suggesting that different pathways can influence disease progression [38].

Paik et al. [39] used a different approach to show the clinical utility of the OncotypeDx classifier of prognosis for node-negative, ER-positive patients who received tamoxifen following local therapy for primary breast cancer. It analyses the expression of a panel of 21 genes, including ER mRNA, downstream ER-regulated genes, HER2 and proliferation-related gene expression levels, which can help in the diagnosis of ER-positive breast cancer that can be treated with tamoxifen [39].

These studies show that the molecular classification of breast cancer may have an impact on the prognosis and prediction, and provide further insights into tumour biology, providing information to both clinicians and scientists. The molecular signatures that define particular groups may lead to the discovery of new therapeutic targets and treatments that are effective in particular molecular subsets.

\section{The power of joint analysis of microarray datasets: meta-analysis}

The extensive use of DNA microarray technology in the characterization of the cell transcriptome is leading to an ever-increasing amount of microarray data from cancer studies. Different datasets for the same type of cancers are available from different microarray studies and this allows the researchers to carry out a more comprehensive analysis of their existing dataset. Besides individual microarrays, meta-analysis can be used to gather and process the datasets from multiple cancer types to investigate common molecular pathways [40-42].

Microarray datasets can be obtained from various public gene expression data repositories including the Stanford Microarray Database (SMD) [43], the National Cancer Institute's Gene Expression Omnibus (GEO) [44] and Oncomine [45]. These databases enable researchers to retrieve and perform analyses on various microarray experiments from different laboratories.

Since all cancer cells share some common characteristics such as loss of growth control, invasion and metastasis, it is very important to identify universal cancer type-independent signatures to better understand cancer pathogenesis and ultimately to improve therapeutic options. Rhodes et al. applied the meta-analysis approach to 21 published cancer microarray datasets, spanning 12 distinct cancer types and identified a set of 67 genes that are universally activated relative to corresponding normal tissues in most cancer types [40].

Collection of independent microarray datasets generated with the common objective of identifying differentially expressed genes in a certain type of cancer has also been performed for breast cancer [46-48]. These types of studies have resulted in the identification of gene sets with a high diagnostic value. In a microarray study with invasive ductal carcinoma samples, a reliable set of 10 genes were identified that can be used as a diagnostic tool for accurate determination of ER status and to make a decision regarding the endocrine therapeutic strategies for breast cancer. The robustness and reliability of these classifiers was confirmed after further testing on three independent microarray gene expression datasets [49].

Meta-analysis approach can provide novel candidates not present in the existing literature allowing reports of multiple genes when neither dataset can report them when analysed individually $[50,51]$.

\section{Large-scale real-time quantitative reverse transcription PCR (qRT-PCR)}

Microarray studies allow high-throughput analysis of expression for thousands of genes and add valuable data to tumour studies. However, once cancer target genes have been identified through this technology, validation of existing microarray data becomes inevitable.

qRT-PCR, also known as real-time PCR, plays an increasingly important role in high-throughput testing of existing microarray data [52]. qRT-PCR is an accurate and sensitive method quantifying mRNA transcripts that uses the quantitative relationship between the amount of starting target sample 
and the amount of PCR product at any given PCR cycle number. The method allows the detection of amplicon accumulation since it is performed using sensitive fluorogenic Taq-Man Probes, molecular beacons, and scorpions or more sensitive but less specific intercalating dyes like SYBR Green I which only fluoresce intensely when associated with double-stranded DNA [53]. The amount of fluorescence produced from the fluorogenic probes is measured at each amplification cycle. qRT-PCR has the advantages of requiring smaller quantities of sample and producing fast, accurate and easily reproducible quantitative results with little manipulation of the samples [54].

qRT-PCR is attractive for clinical use since it can be automated and performed on fresh or archived formalin-fixed, paraffin-embedded (FFPE) tissue samples [55, 56]. The biological classification formed using microarray data has been validated with freshly frozen breast tissues from multiple patient cohorts by qRT-PCR. Sorlie et al. [57] validated and characterized two previously defined clinically relevant subtypes of early stage breast carcinomas, luminal $\mathrm{A}$ and basal-like, by using three different microarray platforms. The set of 54 predictor genes identified in this study were validated by qRT-PCR using the RNA isolated from the same fresh frozen breast tumour samples that were used in microarray platforms. These genes were defined as potential prognostic molecular markers for these subtypes of breast cancer [57]. Perreard et al. [58] used the power of qRT-PCR to make the clinical distinction between ER-positive and ER-negative breast tumours and identified additional subtypes of breast tumours that have prognostic value. In another study, the results obtained with a 70 -gene expression profile described previously in breast cancer [25] were reproduced with qRT-PCR by using a different set of frozen breast cancer samples [59]. Urban et al. [60] used two different microarray platforms and qRT-PCR in their study and identified the UPA gene associated with distant metastasis-free survival in ErbB2-positive breast tumours that can be used as a powerful prognostic indicator.

Recently, an exhaustive analysis of popular microarray platforms by the multi-centre consortium, the MicroArray Quality Control (MAQC) consortium, delivered reassuringly impressive results for the accuracy and reproducibility of commonly used microarray platforms [61]. The focus on their study has been the identification of common transcripts that are mutually represented among the various microarray platforms included in the analysis. Based on the MAQC dataset, Canales et al. [55] used three different RT-PCR methods to profile the same RNA samples to determine the concordance between the microarray-based measurements and RT-PCR results. They found that the correlation coefficients were very high for several hundred genes examined with both methods.

FFPE tissue samples are well-suited for qRTPCR expression studies [62]. It has been shown that it is feasible to extract and purify RNA from FFPE tissue and to perform gene expression experiments although fragmentation of RNA can occur during the fixation process [56]. Retrospective clinical studies generally use FFPE tissue, as it is the most widely available material. These tissues have been used extensively and provide a major resource for understanding disease mechanisms and using the power of differentially expressed genes to evaluate possible new diagnostic or therapeutic approaches.

A diagnostic assay, OncotypeDX ${ }^{\mathrm{TM}}$ (Genomic Health Inc., Redwood City, CA, USA), has been developed as an RT-PCR-based assay performed with FFPE tumour tissue. It analyses the expression of a panel of 21 genes, which can help in the diagnosis of ER-positive breast cancer [39]. The genes identifying molecular subtypes of breast cancer with prognostic significance obtained from microarrays with fresh-frozen tissues were also used to diagnose biological subtypes of breast cancer in FFPE tissues by qRT-PCR. The subtype classifications of the breast tumour with the diagnostic gene set were highly comparable between FF and FFPE tissue samples [63]. Collectively, these studies show the reproducibility of microarray data with the qRTPCR technique.

Although limited to quantification of mRNA transcripts, the sensitivity, reproducibility, expandability and cost-effectiveness of qRT-PCR make it a benchmark technology for integration with microarray technology.

\section{CONCLUDING REMARKS}

Recent advances in genomics and genomic technologies have made it possible to study cancer in many novel ways. Microarray technology has been used to study all aspects of cancer biology that help 
to uncover the molecular mechanism of cancer development and has an impact on diagnosis, prognosis, drug responses and new therapeutic approaches in cancer. Another important aspect in cancer studies is establishing the epigenetic profile of a cancer type, since modification of proteins associated with chromatin and methylation of $\mathrm{CpG}$ sites in the DNA has a profound effect on gene expression. Such studies defining methylation signature could not only help staging of cancer cases but also help to identify the potential molecular markers for early cancer detection, assess cancer risk and improve monitoring of cancer prognosis. New genome-wide, high-throughput tools, such as Chromatin-immunoprecipitation (ChIP)-on-microarray (or ChIP-Chip) are also becoming very useful for studying epigenetic modifications in cells. ChIP combined with highresolution microarray analysis allows the examination of genome-wide nucleosome occupancy and histone modification status [64]. Genome-wide chromatin status can then be compared with global gene expression patterns to reveal connections between specific patterns of histone modifications and the resulting gene expression in the normal or malignant phenotype of a cell. Genomic studies examining tumour sets with multiple complementary technologies, including comparative genomic hybridization (CGH), single nucleotide polymorphisms (SNPs), serial analysis of gene expression (SAGE), ChIP-chip data, proteomics and gene expression array can provide a multitude of opportunities for cancer research. The large amount of discoveries by these high-throughput techniques could then be integrated with emerging bioinformatics to increase our knowledge in cancer development. Combining the results of these multidisiplinary approaches will contribute to a better biological understanding of, and, therefore, to the improvement of the clinical management of cancer.

\section{Key Points}

- Gene expression profiling of breast tumours can distinguish subtypes of breast cancer and has an impact on the diagnosis, prognosis and treatment of the disease when combined with clinical data.

- Meta-analysis uses the power of combining different microarray datasets to identify common molecular signatures among individual or multiple cancer types.

- qRT-PCR can be used to validate gene expression profiles and performed on both fresh or FFPE tissue samples.

\section{Acknowledgements}

We would like to thank Bilkent University and the Scientific and Technical Research Council of Turkey for supporting work in our laboratory.

\section{References}

1. Zhao X, Li C, Paez JG, et al. An integrated view of copy number and allelic alterations in the cancer genome using single nucleotide polymorphism arrays. Cancer Res 2004;64: 3060-71.

2. Bignell GR, Huang J, Greshock J, et al. High-resolution analysis of DNA copy number using oligonucleotidemicroarrays. Genome Res 2004;14:287-95.

3. Pinkel D, Segraves R, Sudar D, et al. High resolution analysis of DNA copy number variation using comparative genomic hybridization to microarrays. Nat Genet 1998;20: 207-11.

4. Atalay A, Crook T, Ozturk M, et al. Identification of genes induced by BRCA1 in breast cancer cells. Biochem Biophys Res Commun 2002;299:839-46.

5. Porter DA, Krop IE, Nasser S, et al. A SAGE (serial analysis of gene expression) view of breast tumor progression. Cancer Res 2001;61:5697-702.

6. Allinen M, Beroukhim R, Cai L, et al. Molecular characterization of the tumor microenvironment in breast cancer. Cancer Cell 2004;6:17-32.

7. Kolch W, Mischak H, Pitt AR. The molecular make-up of a tumour: proteomics in cancer research. Clinical Sci 2005; 108:369-83.

8. Fuchs F, Boutros M. Cellular phenotyping by RNAi. Brief Funct Genomic Proteomic 2006;5:52-6.

9. Molloy MP, Witzmann AF. Proteomics: technologies and applications. Brief Funct Genomic Proteomic 2002;1:23-39.

10. Claudino WM, Quattrone A, Biganzoli L, et al. Metabolomics: available results, current research projects in breast cancer, and future applications. J Clin Oncol 2007; 25:2840-6.

11. Hanauer DA, Rhodes DR, Sinha-Kumar C, et al. Bioinformatics approaches in the study of cancer. Curr Mol Med 2007;7:133-41.

12. Dhanasekaran SM, Barrette TR, Ghosh D, etal. Delineation of prognostic biomarkers in prostate cancer. Nature 2001; 412:822-6.

13. Chen X, Cheung ST, So S, et al. Gene expression patterns in human liver cancers. Mol Biol Cell 2002;13:1929-39.

14. Luo J, Duggan DJ, Chen Y, et al. Human prostate cancer and benign prostatic hyperplasia: molecular dissection by gene expression profiling. Cancer Res 2001;61:4683-8.

15. Iacobuzio-Donahue CA, Maitra A, Olsen $\mathrm{M}$, et al. Exploration of global gene expression patterns in pancreatic adenocarcinoma using cDNA microarrays. AmJ Pathol 2003;162:1151-62.

16. Higgins JPT, Shinghal R, Gill H, et al. Gene expression patterns in renal cell carcinoma assessed by complementary DNA microarray. AmJ Pathol 2003;162:925-32.

17. Bittner M, Meltzer P, Chen Y, et al. Molecular classification of cutaneous malignant melanoma by gene expression profiling. Nature 2000;406:536-40. 
18. Xu L, Tan AC, Naiman DQ, et al. Robust prostate cancer marker genes emerge from direct integration of inter-study microarray data. Bioinformatics 2005;21:3905-11.

19. Badve S, Turbin D, Thorat MA, et al. FOXA1 expression in breast cancer-correlation with luminal subtype $A$ and survival. Clin Cancer Res 2007;13:4415-21.

20. Sotiriou C, Wirapati P, Loi S, et al. Gene expression profiling in breast cancer: understanding the molecular basis of histologic grade to improve prognosis. J Natl Cancer Inst 2006;98:262-72.

21. Loi S, Haibe-Kains B, Desmedt C, et al. Definition of clinically distinct molecular subtypes in estrogen receptorpositive breast carcinomas through genomic grade. J Clin Oncol 2007;25:1239-46.

22. Weigelt $\mathrm{B}, \mathrm{Hu} \mathrm{Z}, \mathrm{He} \mathrm{X}$, et al. Molecular portraits and 70 -gene prognosis signature are preserved throughout the metastatic process of breast cancer. Cancer Res 2005;65: 9155-8.

23. Nielsen TO, Hsu FD, Jensen K, et al. Immunohistochemical and clinical characterization of the basal-like subtype of invasive breast carcinoma. Clinl Cancer Res 2004;10: 5367-74.

24. Sorlie T, Perou CM, Tibshirani R, et al. Gene expression patterns of breast carcinomas distinguish tumor subclasses with clinical implications. Proc Natl Acad Sci USA 2001;98: 10869-74.

25. van't Veer LJ, Dai $H$, van de Vijver MJ, et al. Gene expression profiling predicts clinical outcome of breast cancer. Nature (London) 2002;415:530-6.

26. van de Vijver MJ, He YD, van't Veer LJ, et al. A geneexpression signature as a predictor of survival in breast cancer. NEnglJ Med 2002;347:1999-2009.

27. Ahr A, Karn T, Solbach C, et al. Identification of high risk breast-cancer patients by gene expression profiling. Lancet 2002;359:131-2.

28. Sotiriou C, Neo SY, McShane LM, et al. Breast cancer classification and prognosis based on gene expression profiles from a population-based study. Proc Natl Acad Sci USA 2003; 100:10393-8.

29. Huang E, Cheng SH, Dressman H, et al. Gene expression predictors of breast cancer outcomes. Lancet 2003;361: 1590-6.

30. Cleator S, Ashworth A. Molecular profiling of breast cancer: clinical implications. BrJ Cancer 2004;90:1120-4.

31. Gruvberger S, Ringner M, Chen Y, et al. Estrogen receptor status in breast cancer is associated with remarkably distinct gene expression patterns. Cancer Res 2001;61:5979-84.

32. West M, Blanchette C, Dressman $\mathrm{H}$, et al. Predicting the clinical status of human breast cancer by using gene expression profiles. Proc Natl Acad Sci USA 2001;98: $11462-7$

33. Perou CM, Sorlie T, Eisen MB, et al. Molecular portraits of human breast tumours. Nature 2000;406:742-52.

34. Sorlie T, Tibshirani R, Parker J, et al. Repeated observation of breast tumor subtypes in independent gene expression data sets. Proc Natl Acad Sci USA 2003;100:8418-23.

35. Pusztai L, Ayers M, Stec J, et al. Gene expression profiles obtained from fine-needle aspirations of breast cancer reliably identify routine prognostic markers and reveal large-scale molecular differences between estrogen-negative and estrogen-positive tumors. Clin Cancer Res 2003;9: 2406-15

36. Rouzier R, Perou CM, Symmans WF, et al. Breast cancer molecular subtypes respond differently to preoperative chemotherapy. Clin Cancer Res 2005;11:5678-85.

37. Buyse M, Loi S, Van't Veer L, et al. Validation and clinical utility of a 70-Gene prognostic signature for women with node-negative breast cancer. J Natl Cancer Inst 2006;98: 1183-92.

38. Wang Y, Klijn JGM, Zhang Y, et al. Gene-expression profiles to predict distant metastasis of lymph-node-negative primary breast cancer. Lancet 2005;365:671.

39. Paik S, Shak S, Tang G, et al. A multigene assay to predict recurrence of tamoxifen-treated, node-negative breast cancer. NEngl J Med 2004;351:2817-26.

40. Rhodes DR, Yu J, Shanker K, et al. Large-scale metaanalysis of cancer microarray data identifies common transcriptional profiles of neoplastic transformation and progression. Proc Natl Acad Sci USA 2004;101:9309-14.

41. Choi H, Shen R, Chinnaiyan AM, et al. A latent variable approach for meta-analysis of gene expression data from multiple microarray experiments. BMC Bioinformatics 2007; 8:364.

42. Xu L, Geman D, Winslow RL. Large-scale integration of cancer microarray data identifies a robust common cancer signature. BMC Bioinformatics 2007;8:275.

43. Sherlock G, Hernandez-Boussard T, Kasarskis A, et al. The Stanford microarray database. Nucleic Acids Res 2001;29: $152-5$.

44. Barrett T, Suzek TO, Troup DB, et al. NCBI GEO: mining millions of expression profiles-database and tools. Nucleic Acids Res 2005;33:D562-6.

45. Rhodes DR, Yu J, Shanker K, etal. ONCOMINE: a cancer microarray database and integrated data-mining platform. Neoplasia 2004;6:1-6.

46. Rhodes DR, Barrette TR, Rubin MA, et al. Meta-analysis of microarrays: interstudy validation of gene expression profiles reveals pathway dysregulation in prostate cancer. Cancer Res 2002;62:4427-33.

47. Jiang H, Deng Y, Chen HS, et al. Joint analysis of two microarray gene-expression data sets to select lung adenocarcinoma marker genes. BMC Bioinformatics 2004;5:81.

48. Shen D, He J, Chang HR. In silico identification of breast cancer genes by combined multiple high throughput analyses. Int J Mol Med 2005;15:205-12.

49. Schneider J, Ruschhaupt M, Buness A, et al. Identification and meta-analysis of a small gene expression signature for the diagnosis of estrogen receptor status in invasive ductal breast cancer. IntJ Cancer 2006;119:2974-9.

50. Choi JK, Choi JY, Kim DG, et al. Integrative analysis of multiple gene expression profiles applied to liver cancer study. FEBS Lett 2004;565:93-100.

51. Grutzmann R, Boriss $\mathrm{H}$, Ammerpohl O, et al. Metaanalysis of microarray data on pancreatic cancer defines a set of commonly dysregulated genes. Oncogene 2005;24: 5079-88.

52. Bernard PS, Wittwer CT. Real-time PCR technology for cancer diagnostics. Clin Chem 2002;48:1178-85. 
53. Peters IR, Helps CR, Hall EJ, et al. Real-time RT-PCR: considerations for efficient and sensitive assay design. J Immunol Methods 2004;286:203-17.

54. Bustin SA. Quantification of mRNA using real-time reverse transcription PCR (RT-PCR): trends and problems. J Mol Endocrinol 2002;29:23-39.

55. Canales RD, Luo Y, Willey JC, et al. Evaluation of DNA microarray results with quantitative gene expression platforms. Nat Biotechnol 2006;24:1123-31.

56. Specht K, Richter T, Muller U, et al. Quantitative gene expression analysis in microdissected archival tissue by real-time RT-PCR. J Mol Med 2000;78:B27.

57. Sorlie $\mathrm{T}$, Wang $\mathrm{Y}$, Xiao C, et al. Distinct molecular mechanisms underlying clinically relevant subtypes of breast cancer: gene expression analyses across three different platforms. BMC Genomics 2006;7:127-41.

58. Perreard L, Fan C, Quackenbush JF, et al. Classification and risk stratification of invasive breast carcinomas using a real-time quantitative RT-PCR assay. Breast Cancer Res 2006;8:R23.

59. Espinosa E, Vara JA, Redondo A, et al. Breast cancer prognosis determined by gene expression profiling: a quantitative reverse transcriptase polymerase chain reaction study. J Clin Oncol 2005;23:7278-85.

60. Urban $\mathrm{P}$, Vuaroqueaux $\mathrm{V}$, Labuhn $\mathrm{M}$, et al. Increased expression of urokinase-type plasminogen activator mRNA determines adverse prognosis in ErbB2-positive primary breast cancer. J Clin Oncol 2006;24:4245-53.

61. MAQC Consortium. The microarray quality control (MAQC) project shows inter- and intraplatform reproducibility of gene expression measurements. Nat Biotechnol 2006;24:1151-61.

62. Ma XJ, Patel R, Wang X, et al. Molecular classification of human cancers using a 92-gene real-time quantitative polymerase chain reaction assay. Arch Pathol Lab Med 2006; 130:465-73.

63. Mullins M, Perreard L, Quackenbush JF, et al. Agreement in breast cancer classification between microarray and quantitative reverse transcription PCR from fresh-frozen and formalin-fixed, paraffin-embedded tissues. Clin Chem 2007;53:1273-9.

64. Kim TH, Ren B. Genome-wide analysis of proteinDNA interactions. Annu Rev Genomics Hum Genet 2006;7: 81-102. 